\title{
Hydrogen-Rich Water Ameliorates Murine Chronic Graft-versus- Host Disease through Antioxidation
}

\author{
Liren Qian $(\mathbb{D}$, Jiaxin Liu, Weina Ma, Yu Liu, Xiaona Wang, and Daihong Liu \\ Senior Department of Hematology, The Fifth Medical Center, Chinese PLA General Hospital, Chinese PLA Medical School, \\ Beijing, China \\ Correspondence should be addressed to Daihong Liu; daihongrm@163.com
}

Received 7 August 2021; Accepted 25 September 2021; Published 14 October 2021

Academic Editor: Ivan Srejovic

Copyright (C) 2021 Liren Qian et al. This is an open access article distributed under the Creative Commons Attribution License, which permits unrestricted use, distribution, and reproduction in any medium, provided the original work is properly cited.

\begin{abstract}
Background. Allogeneic hematopoietic stem cell transplantation (allo-HSCT) is an important treatment option for various hematopoietic diseases and certain hereditary diseases. Chronic graft-versus-host disease (cGVHD) has become the main lifethreatening complication and cause of death in later stage postallo-HSCT. Current treatment options for cGVHD are limited. Hydrogen gas $\left(\mathrm{H}_{2}\right)$ has been demonstrated that has antioxidative, anti-inflammatory, and antifibrosis effects. The aim of this study was to confirm whether oral administration hydrogen-rich water exerted therapeutic effects on a scleroderma cGVHD mouse model and tried to explain the mechanism underly it. Methods. A mouse cGVHD model was established by haploidentical bone marrow transplantation. To evaluate therapeutic effects of $\mathrm{H}_{2}$ on cGVHD, survival rate, changes in clinical scores, and skin pathologic characteristics of cGVHD mice were observed. To evaluate its therapeutic mechanism, we detected the expression levels of antioxidative enzymes heme oxygenase-1(HO-1) and $\mathrm{NAD}(\mathrm{P}) \mathrm{H}$ : quinone acceptor oxidoreductase 1(NQO1) in skin homogenates. We also detected the expression level of the apoptotic protein caspase-3 in skin homogenates. Results. 1-month survival rate of cGVHD mice in the hydrogen group reached $93.3 \%$, significantly higher than $66.7 \%$ in the nonhydrogen group $(p<0.05)$. Clinical score of cGVHD mice was improved by hydrogen-rich water at 96 days posttransplantation $(2.2$ versus $4.5, p<0.05)$. The skin pathological condition of cGVHD mice was significantly improved by hydrogen-rich water. At 96 days posttransplantation, average skin pathological hematoxylin and eosin (HE) staining score in the hydrogen group was 1.05 , which was significantly lower than 3.2 in the nonhydrogen group $(p<0.01)$. Average Masson staining score was 0.6 point in the hydrogen group, lower than 0.9 point in the nonhydrogen group $(p<0.05)$. Both the relative expression levels of HO-1 and NQO1 proteins in skin specimens of cGVHD mice in the hydrogen group were lower than that in the nonhydrogen group (2.47 versus 6.21 and 1.83 versus $3.59, p<0.05$ ). The relative expression level of caspase-3 protein in skin specimens of cGVHD mice increased to 7.17 on the 96th day after transplantation, significantly higher than 4.36 in the hydrogen group. Conclusion. In this study, we found that oral hydrogen-rich water improved the survival rate and clinical symptoms of cGVHD mice by antioxidant and antiapoptosis. This study would pave the way for further clinical study, which may provide a new treatment option for cGVHD.
\end{abstract}

\section{Introduction}

Allogeneic hematopoietic stem cell transplantation (alloHSCT) is an important treatment option for various hematopoietic diseases and certain hereditary diseases. Chronic graft-versus-host disease (cGVHD) has become the main life-threatening complication and cause of death in later stage postallo-HSCT $[1,2]$. With the decrease in early mortality posttransplantation, the increasing upper age limit of recipients, and the widespread application of unrelated donors and peripheral blood hematopoietic stem cells, the incidence of cGVHD has gradually increased [3, 4]. 2-year cumulative incidence of cGVHD posttransplantation that needs treatment was 30\%-40\% [2]. Glucocorticoids with or without calcineurin inhibitors (CNI) were always used as the initial treatment of cGVHD in the past few decades [2]. However, glucocorticoids may cause serious adverse effects after long-term application, including severe infections, peptic ulcers, femoral head necrosis, osteoporosis, diabetes, and hypertension [5]. Besides, during the tapering of 
glucocorticoids, cGVHD symptoms often relapse or even aggravate [5]. If the disease relapses or progresses, secondline treatment is often required. 50\%-60\% cGVHD patients need second-line therapy $[6,7]$. However, there is currently no standard preferred second-line treatment [8]. Although some new drugs such as ruxolitinib have brought unprecedented curative effects in this field in recent years [9-11], clinical trials are still encouraged, and a better therapy method is in urgent need for cGVHD [12].

In 2007, Ohsawa et al. systematically confirmed the free radicals scavenging ability of hydrogen [13]. They found that $\mathrm{H}_{2}$ had similar therapeutic effects in a cerebral ischemiareperfusion injury rat model by scavenging hydroxyl radicals (.OH) comparing with tacrolimus. Besides to its free radical scavenging ability, researchers also found that hydrogen has anti-inflammatory effects in autoimmune hepatitis [14], systemic inflammatory response syndrome [15], inflammatory bowel disease [16], allergic dermatitis [17], lipopolysaccharide- (LPS-) induced paw edema [18], and other animal inflammatory disease models, which is also similar to tacrolimus. Moreover, $\mathrm{H}_{2}$ has antifibrosis effects. It was found that breathing $4 \% \mathrm{H}_{2}$ significantly delayed the progression of pulmonary fibrosis in a radiation induced pulmonary fibrosis model [19]. They confirmed that $\mathrm{H}_{2}$ significantly reduced the fibrotic lesions in the lungs of mice. The main pathophysiological process of cGVHD is immunoinflammatory responses, and the characteristic pathological change is fibrosis $[12,20,21]$. Oxidative stress, inflammation imbalance, and fibrosis play important roles in the progression of cGVHD $[12,20,21]$. Therefore, we speculated that $\mathrm{H}_{2}$ may exert potential therapeutic effects on cGVHD after allo-HSCT. In this study, an attempt was made to confirm whether oral administration hydrogen-rich water exerted therapeutic effects on a scleroderma cGVHD mouse model and tried to explain the mechanism underly it.

\section{Materials and Methods}

2.1. Hydrogen-Rich Water Production. Hydrogen-rich water was produced by dissolving hydrogen in sterile drinking water for 6 hours under high pressure $(0.4 \mathrm{MPa})$ to a supersaturated level as we previously reported [22-24]. Hydrogen-rich water was freshly prepared every 12 hours, which ensured that a concentration of more than $0.6 \mathrm{mmol} / \mathrm{L}$ was maintained. Gas chromatography (Biogas Analyzer Systems-1000, Mitleben, Japan) was used to confirm the content of hydrogen in saline by the method described by Ohsawa et al. [13].

2.2. Mice. All the protocols were approved by the Chinese PLA General Hospital in accordance with the Guide for Care and Use of Laboratory Animals published by the US NIH (publication No. 96-01). Female C57BL/6 N mice and male B6D2F1 mice were obtained from Beijing Vital River Laboratory Animal, Inc. (Beijing, China, http://www.vitalriver .com.cn). All mice were studied at between 10 and 12 weeks of age. Mice were housed in autoclaved cages with sterile food and water.

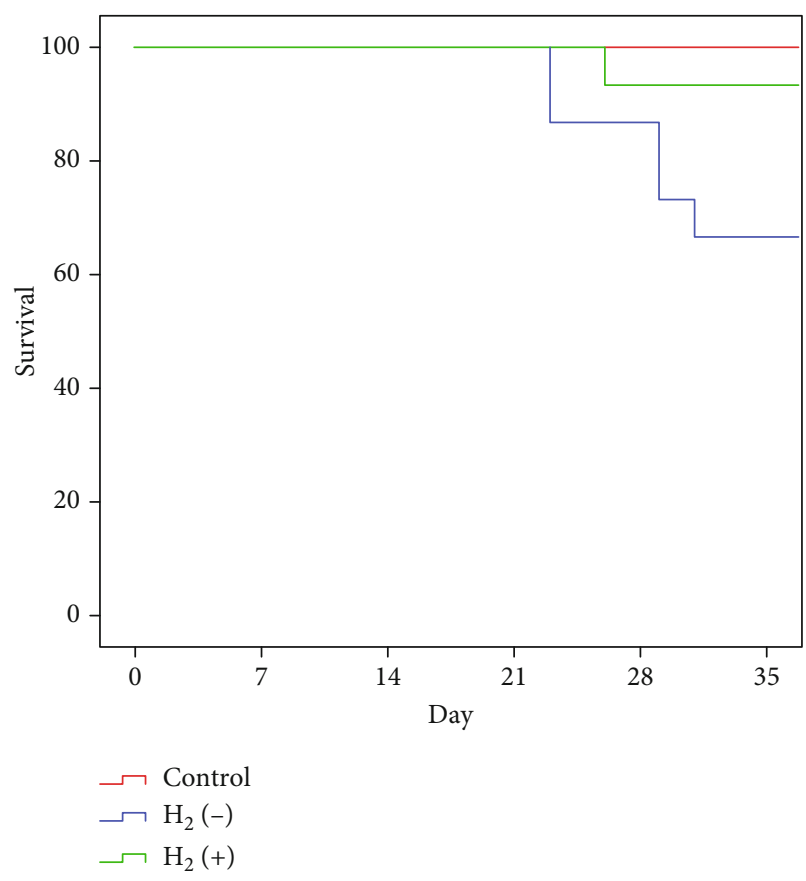

Figure 1: The survival rate of chronic GVHD mice in the hydrogen group was significantly higher than that in the nonhydrogen group $(p<0.05)$.

2.3. Chronic Graft-versus-Host Disease (cGVHD) Model. A cGVHD model was established as previously described [25]. B6D2F1 mice received total body irradiation (TBI) on day 1 ( $5.5 \mathrm{~Gy}$, two doses on the same day, with an interval of 3-4 hours). On day 0 , the control group was injected with $5 \times 10^{6} \mathrm{~T}$ cell-depleted bone marrow cells (TCD-BM) of C57BL/6 mice through the tail vein. In the cGVHD model group, $5 \times 10^{6} \mathrm{TCD}-\mathrm{BM}$ plus purified $1 \times 10^{6}$ splenic $\mathrm{T}$ cells of C57BL/6 mice were injected to irradiated B6D2F1 mice. In the hydrogen group, mice were given hydrogen-rich water from the $8^{\text {th }}$ day posttransplantation when the mice have cGVHD symptoms until they were sacrificed. In the control group and nonhydrogen group, mice were routinely fed with sterile water.

2.4. Survival Assays. After transplantation, the mice were returned to individually ventilated cages and routinely cared. Their survival status was observed daily, and the survival was checked and recorded for 30 days.

2.5. Evaluation of $c G V H D$. Chronic GVHD symptoms of mice are clinically scored every five days after transplantation, mainly from the following 5 aspects [26]: weight loss (scored 0: $<10 \%$; scored 1: $10 \%-25 \%$; scored 2: $>25 \%$ ), activity (scored 0: normal; scored 1: mild to moderately decreased; scored 2: stationary unless stimulated), posture (scored 0: normal; scored 1: hunching only at rest; scored 2: severe hunching impairs movement), fur texture (scored 0 : normal; scored 1: mild to moderate ruffling; scored 2: severe ruffling/poor grooming), and skin integrity (scored 0 : normal; scored 1: incomplete paw/tail scales; scored 2: obvious areas of denuded skin). The scores of these five 


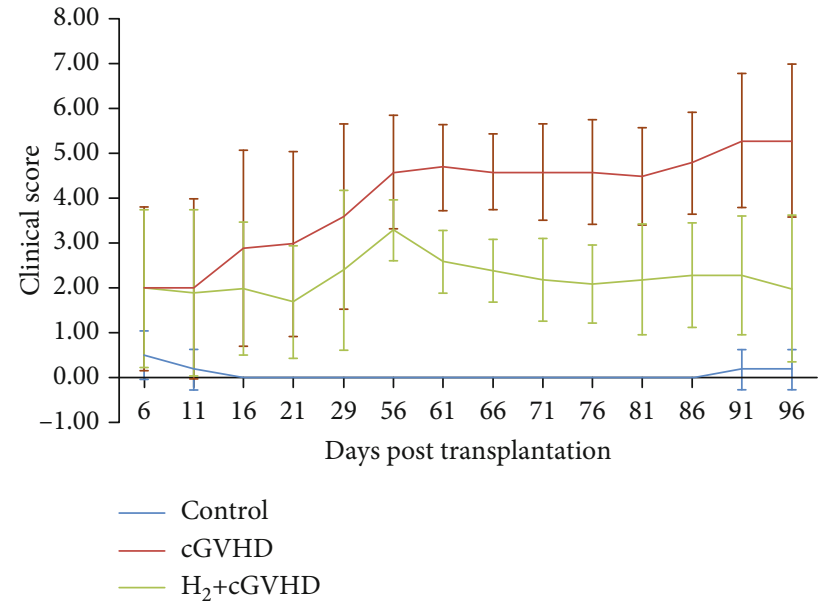

(a)

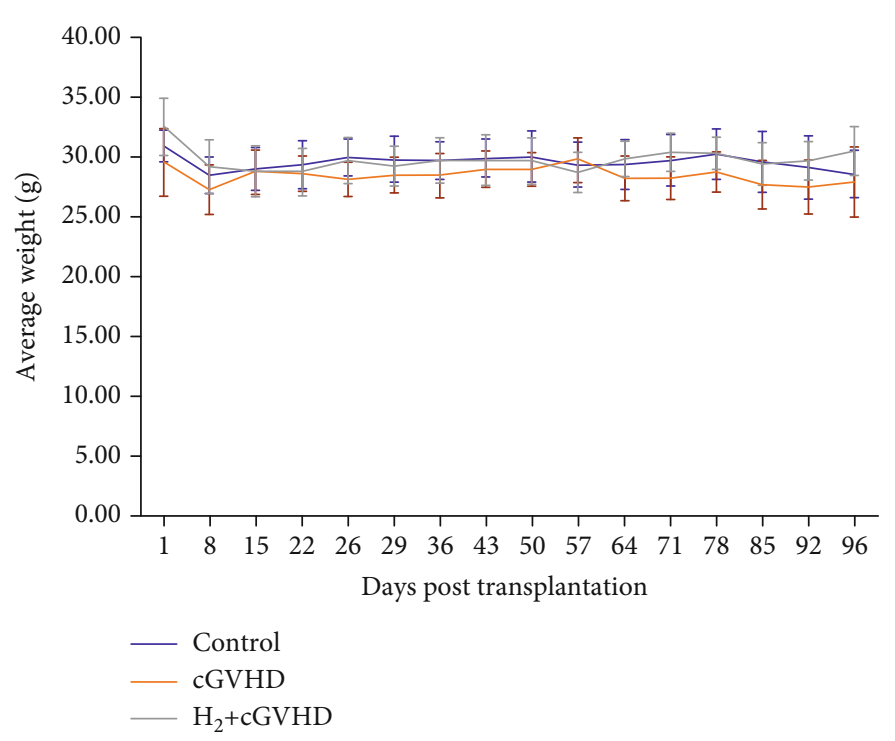

(b)

Figure 2: With the time of drinking $\mathrm{H}_{2}$-rich water increased, the clinical improvement of chronic GVHD mice becomes more obvious. At 96 days after transplantation, the clinical score of chronic GVHD mice in the hydrogen group was significantly lower than that in the nonhydrogen group (Figure 2(a), $p<0.05$ ). The weight of chronic GVHD mice in the hydrogen group was not statistically different from that in the nonhydrogen group (Figure $2(b), p>0.05$ ).

aspects were added together to evaluate the severity of cGVHD.

2.6. Tissue Histopathology. About $2 \mathrm{~cm}^{2}$ shaved skin from interscapular region was selected. The hematoxylin and eosin (HE) staining was performed as we previously described [27]. A dermatologist, blinded to the groups of animals, scored from five aspects: epidermal structural changes, inflammatory cell infiltration, reduction or loss of hair follicles, dermal fibrosis, and reduction or loss of fat. Each index is rated as $0-2$ points according to the severity of the lesion. The total score is between 0 and 10 points [28]. Masson staining was also performed according to the previous literature [23], and scores were given according to the thickness and looseness of collagen fibers: 0 (normal), 0.5 (minor), 1 (mild), 2 (moderate), and 3 (severity). The skin of each group was scored for pathology 96 days after transplantation.

2.7. Western Blot. The specimens of skin tissue were collected and lysed as previously described [29]. The skin samples were collected and frozen in dry ice and stored at $-70^{\circ} \mathrm{C}$ until assayed by WB analysis. We homogenized the skin specimens on ice by sonication and dissolved in lysis buffer, which contains phosphate-buffered saline (PBS, pH 7.4), 1\% Tergitol NP-40 (Sigma-Aldrich, St. Louis, MO), 0.5\% sodium deoxycholate (Sigma), 1\% sodium dodecyl sulfate (SDS) (Sigma), 1 mM EDTA (Sigma), 1 mM EGTA (Sigma), $1 \%$ protease inhibitor cocktail (Sigma), and $0.6 \mathrm{mM}$ phenylmethanesulfonyl fluoride (PMSF). Then, the homogenate was centrifuged at $14,000 \mathrm{rpm}$ for 30 minutes at $4^{\circ} \mathrm{C}$ [30]. Protein concentrations were detected by NanoDrop 1000 spectrophotometer (Thermo Fisher Scientific) [31]. The expression levels of $\mathrm{HO}-1, \mathrm{NQO} 1$, and caspase-3 proteins in the skin tissues of different groups were detected by western blot analysis as previously described [32]. In the western blot analysis, we obtained the following antibodies from Cell Signaling Technology: anti-HO-1, anti-NQO1, anti-caspase3 , and anti- $\beta$-actin.

\section{Results}

\subsection{Therapeutic Effects of Hydrogen on $c G V H D$ Mice}

3.1.1. Hydrogen Increased the Survival Rate of cGVHD Mice. Oral giving more than 2 weeks of hydrogen-rich water improved the survival rate of cGVHD mice (Figure 1). The 30 -day survival rate of cGVHD mice in the hydrogen water group was $93.3 \%$, significantly higher than that in the nonhydrogen cGVHD group $(66.7 \%, p<0.05)$.

3.1.2. Hydrogesgen Improved $c G V H D$ Mice Clinical Symptoms. Compared with the nonhydrogen group, the clinical symptoms of the mice in the hydrogen group began to improve after drinking hydrogen-rich water for one week (Figure 2(a)). With the time of drinking hydrogen-rich water increased, the improvement of clinical symptoms becomes more obvious. At 96 days posttransplantation, average clinical score of the cGVHD mice in the hydrogen group was 2.0 points, which was less than that in the nonhydrogen water group (5.3 points, $p<0.05)$. The average body weight of cGVHD mice in the hydrogen group was higher compared with that of the nonhydrogen water group without statistical difference (30.50 g vs. $27.92 \mathrm{~g}, p>0.05$, Figure 2(b)).

3.1.3. Hydrogen Improved cGVHD Mice Skin Pathology. The skin pathological condition of cGVHD mice has been significantly improved after given hydrogen-rich water. At 96 days posttransplantation, the average skin pathological HE 

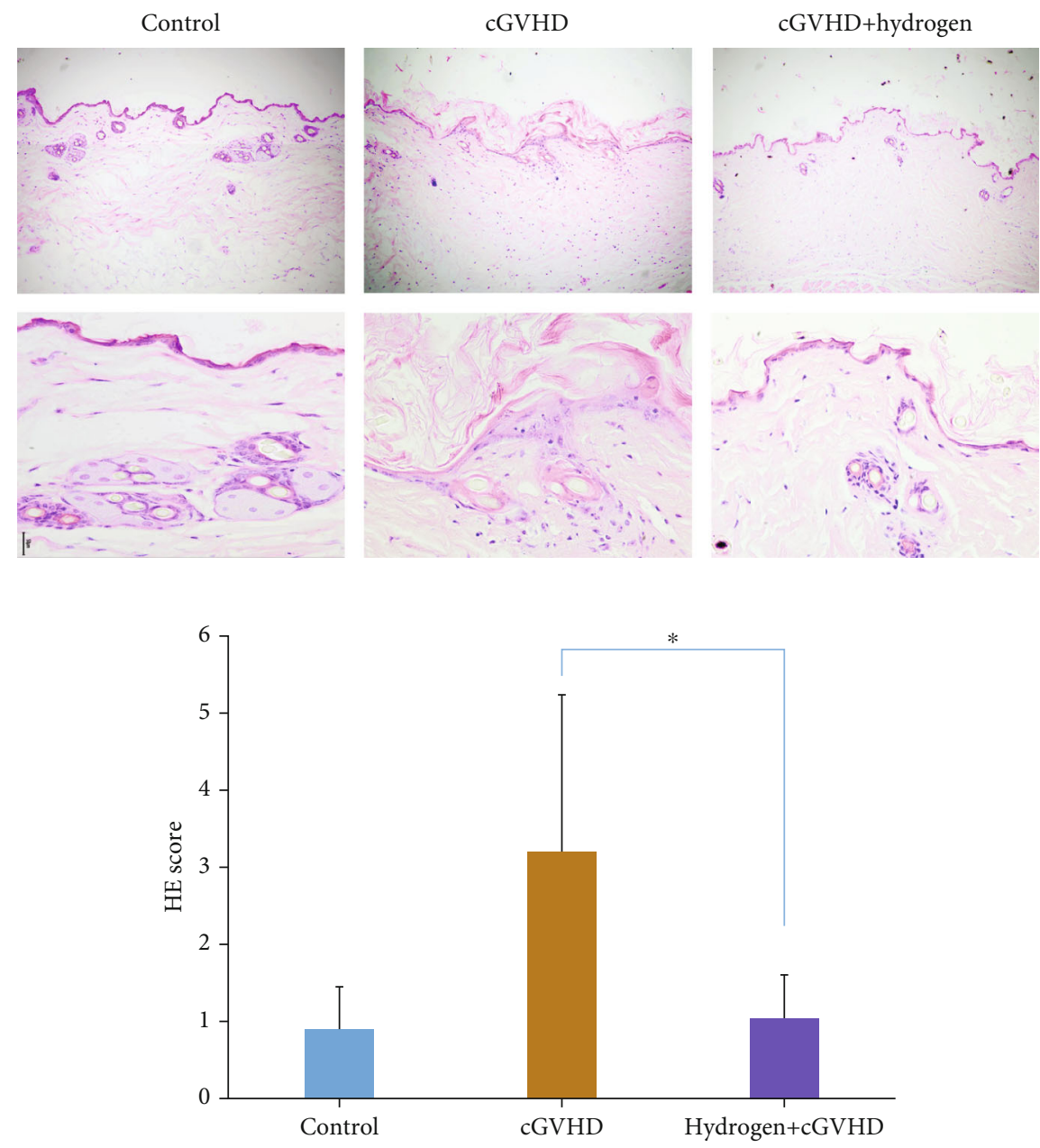

FIgURE 3: The skin pathological HE staining score of chronic GVHD mice in the hydrogen group was significantly lower than that in the nonhydrogen group $(p<0.01)$.

staining score in the hydrogen group was 1.05 , significantly lower than 3.2 in the nonhydrogen group $(p<0.01$, Figure 3). The average Masson staining score was 0.6 point in the hydrogen group, also lower than 0.9 point in the nonhydrogen group ( $p<0.05$, Figure 4$)$.

\subsection{The Mechanism of Hydrogen on cGVHD Mice}

3.2.1. Hydrogen Reduced the Expression Level of HO-1 and NQO1. The relative expression level of HO-1 protein $(/ \beta-$ actin) in the skin tissue of cGVHD mice in the hydrogen group was 2.47 , which was significantly lower than 6.21 in the skin tissue of the nonhydrogen group cGVHD mice (Figure 5(a)). We found that the relative expression of NQO1 protein $(/ \beta$-actin) in the skin tissue of $\mathrm{cGVHD}$ mice in the hydrogen group was 1.83 , which was significantly lower than 3.59 in the nonhydrogen group (Figure 5(b)).

3.2.2. Hydrogen Reduced the Expression Level of Caspase-3. The relative expression of caspase- 3 protein (/ $\beta$-actin) in the skin tissue of cGVHD mice significantly increased to 7.17 on the 96 th day after transplantation, which was much higher than 4.36 in the hydrogen group, suggesting that molecular hydrogen significantly reduced the relative expression of apoptotic protein, which indicated that hydrogen has antiapoptotic ability by reducing the expression of caspase-3 protein (Figure 6).

\section{Discussion}

To our knowledge, this is the first study that proved oral saturated hydrogen-rich water has therapeutic effects on cGVHD in mice with scleroderma. It was confirmed that it exerted therapeutic effects by antioxidation and antiapoptosis. We demonstrated that hydrogen reduced the expression levels of HO-1 and NQO1 proteins in the cGVHD mice. We consider that hydrogen may neutralize oxygen free radicals and reduce the increased levels of $\mathrm{HO}-1$ and NQO1 proteins caused by reactive oxygen species.

In our study, oral saturated hydrogen-rich water increased the 30-day survival rate of cGVHD mice by nearly $30 \%$. The increase in the survival rate illustrated that hydrogen-rich water has therapeutic effects on mouse cGVHD as a whole. In addition, in this cGVHD animal model, we observed that oral hydrogen-rich water significantly improved the clinical symptoms of cGVHD in mice 


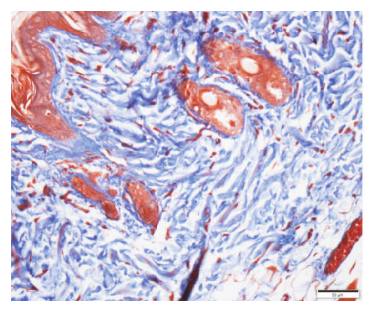

Control

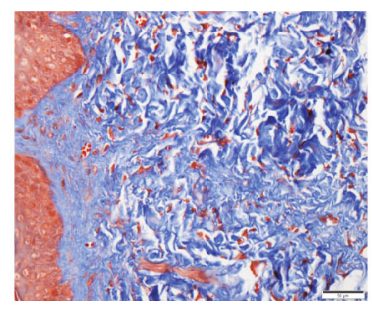

cGVHD

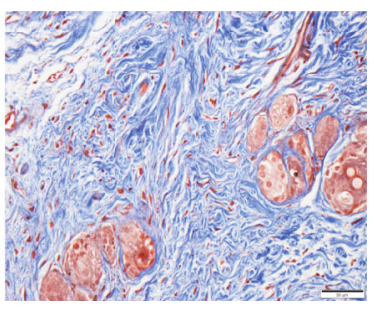

Hydrogen+cGVHD

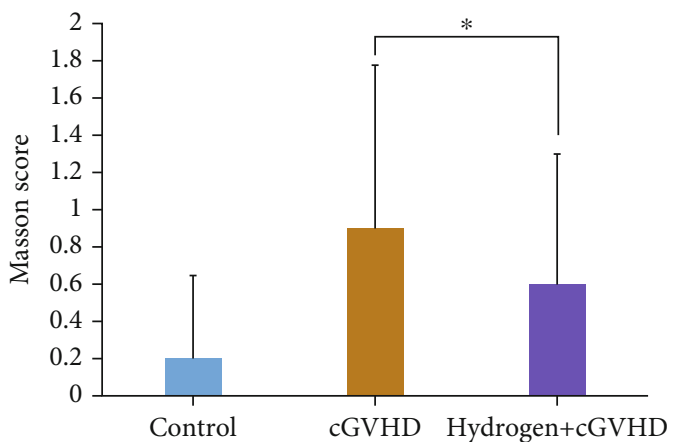

FIgURE 4: The skin pathological Masson staining fibrosis score of chronic GVHD mice in the hydrogen group was significantly lower than that in the nonhydrogen group $(p<0.05)$.
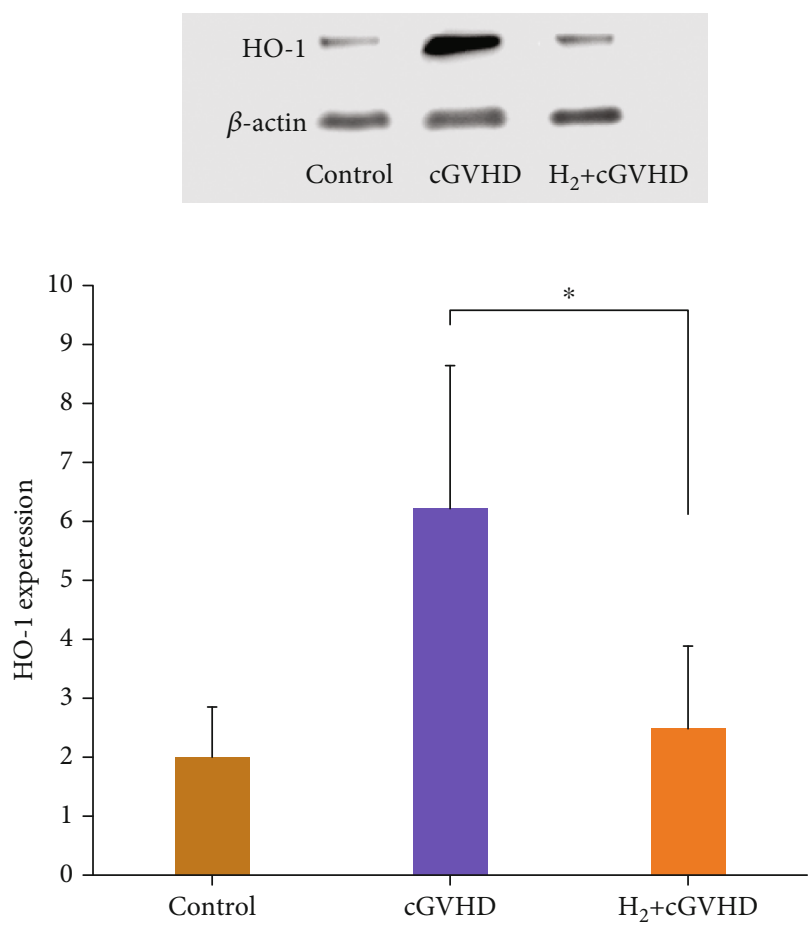

(a)
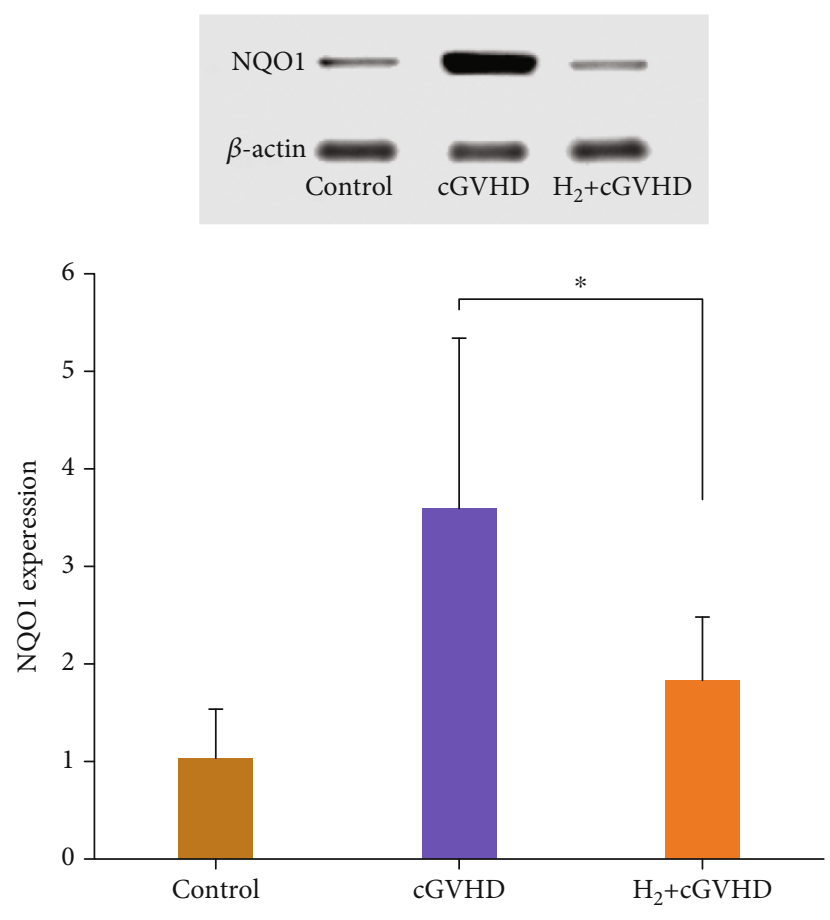

(b)

FIGURE 5: The relative expression level of HO-1 protein in the skin tissue of chronic GVHD mice in the hydrogen group was significantly lower than that in the nonhydrogen group (Figure 5(a), $p<0.05$ ). The relative expression level of NQO1 protein in the skin tissue of chronic GVHD mice in the hydrogen group was significantly lower than that in the nonhydrogen group (Figure $5(\mathrm{~b}), p<0.05$ ).

and improved the skin pathology of mice. Fibrosis has been proven playing an important role in the development of cGVHD disease [19]. Fibrosis leads to organ failure in patients with cGVHD, including scleroderma, bronchitis obliterans, and liver cirrhosis. Our research found that hydrogen-rich water mitigated the degree of skin fibrosis in cGVHD mice and improved the clincial symptoms of scleroderma in cGVHD mice. Formation of fibrosis often requires 


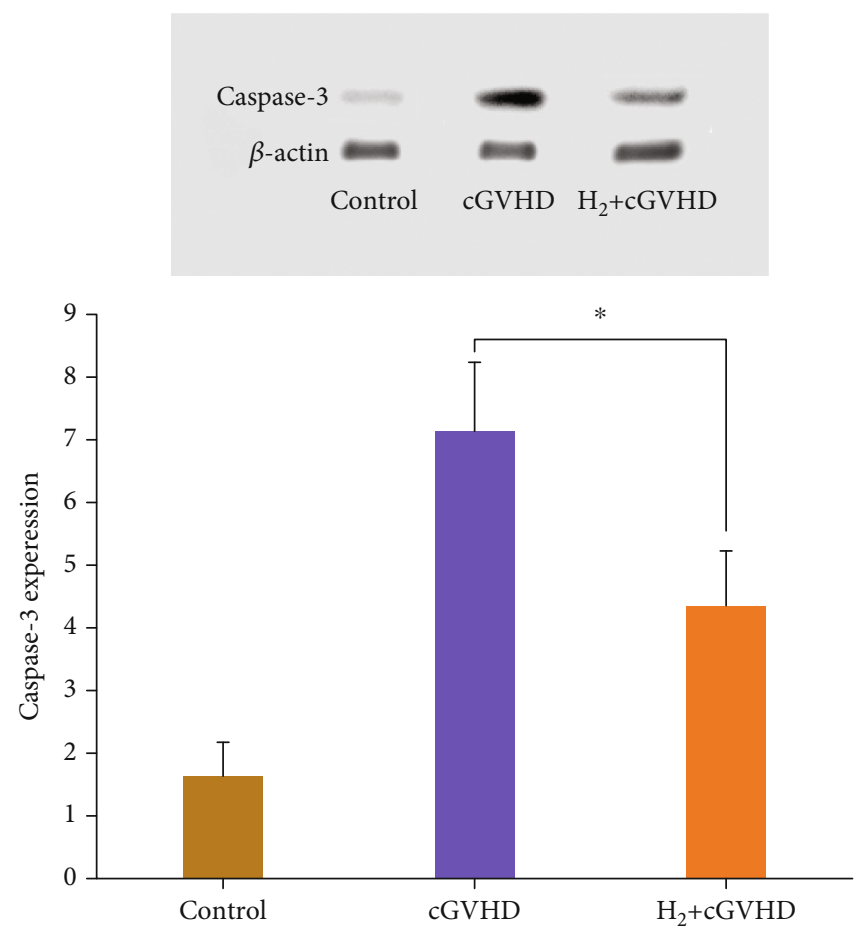

FIgURE 6: The relative expression level of caspase-3 protein in the skin tissue of chronic GVHD mice in the hydrogen group was significantly lower than that in the nonhydrogen group $(p<0.05)$.

a relatively long-term process. Long-term oral hydrogenrich water has no obvious toxic and side effects, making it can be used in a long-term. This feature is adapted to the long-term, repeated, and prolonged disease characteristics of cGVHD, which makes hydrogen-rich water very suitable for cGVHD. Previously, we have demonstrated that the survival rate of another cGVHD mice model was increased by intraperitoneally injecting hydrogen-rich saline, and the pathological changes in skin was also improved [27]. In this study, we used oral hydrogen-rich water, which is more convenient than hydrogen-rich saline for injection and inhalation of hydrogen gas.

HO-1 and NQO1 are two important antioxidant enzymes. HO-1 mainly catalyzes and decomposes heme into ferrous iron, carbon monoxide, and biliverdin and prevents the prooxidation effect of heme. Its byproduct bilirubin and reduced bilirubin exert effective antioxidative ability by scavenging free radicals $[33,34]$. The expression level of HO-1 is significantly positively correlated with the levels of ROS. When the ROS levels in the body increased by various pathological conditions like hypoxia and acidosis, HO-1 would rise rapidly, playing a cytoprotective role in the body [34], which promoted heme catabolism and prevents induction of programmed cell death [35]. NQO1 is a cellprotecting antioxidant enzyme that exerts antioxidant effects from many aspects. NQO1 catalyzed quinone to hydroquinone, promoting the excretion of quinone. It also reduced quinones, quinoneimines, nitroaromatics, and azo dyes, thereby reducing the redox cycle to produce ROS, preventing oxidative damage [36]. Wefers et al. first confirmed that NQO1 has a direct antioxidant effect [37]. It was confirmed that NQO1 relies on the two-electron reduction mechanism, preventing quinone from participating in the oxidation cycle and generating active oxygen. As oxidative stress events occur in the body, NQO1 will also increase and play a protective role. Previously, we have confirmed that hydrogen regulated the levels of antioxidant enzymes superoxide dismutase (SOD), glutathione (GSH), and lipid oxidation product malondialdehyde (MDA) in the peripheral blood of mice injured by irradiation [22], exerting its radioprotective effects. In the current study, we found that in the skin tissues of mice, the expression levels of HO-1 and NQO1 proteins in the hydrogen group were significantly lower than those in the nonhydrogen cGVHD group. Many studies have confirmed that molecular hydrogen can directly react with oxygen free radicals such as hydroxyl radicals, thereby reducing oxidative damage $[13,19]$. We believed that molecular hydrogen may reduce the oxidative stress level in cGVHD mice, thereby reducing the expression levels of HO-1 and NQO1 proteins in CGVHD mice.

Caspase- 3 is one of the executioner caspases in apoptosis. It plays a vital role in cell apoptosis. It is responsible for cleaving most of the currently known apoptosisrelated substrates. At the terminal of apoptosis, it is responsible for decomposing structural and regulatory proteins that shut down cell functions [38]. Chronic GVHD leads to apoptosis of tissue cells, resulting in a series of clinical manifestations. Our research found that the level of apoptosis protein caspase-3 in the molecular hydrogen group was significantly reduced. At present, we still consider molecular hydrogen exerting its antiapoptotic effect through antioxidation. 
This research supplied novel ideas that were for treating cGVHD and has potential clinical application prospects, mainly due to the following points: first, $\mathrm{H}_{2}$ is nontoxic side effects and no residue in the body [39], which is adapted for the long-term, repeated, and prolonged disease characteristics of cGVHD. Second, hydrogen molecules have great penetrating ability because they are very small. They can quickly penetrate biological membranes and reach high concentration in cells to exert therapeutic effects [13]. Third, the price of $\mathrm{H}_{2}$ is low and easy to get. Although the current study confirmed that oral hydrogen-rich water has therapeutic effects on cGVHD and attempted to explain its mechanism, this research was limited confirming its therapeutic effect in a scleroderma cGVHD model. Chronic GVHD often involves multiple organs, including lungs, eyes, joints, gastrointestinal tract, and liver. As for whether hydrogen has therapeutic effects on cGVHD with other organs, it still needs to be further explored. Current studies on $\mathrm{H}_{2}$ have so far been mostly limited to animal or clinical observational researches. Current clear mechanism is its ability of scavenging free radicals. Its antiapoptotic and antifibrosis effects still mainly depended on its ability of scavenging free radicals. As for whether hydrogen gas is a signal molecule, the regulation of HO-1, NQO1, and caspase-3 protein expression levels through signal pathways still needs further research to confirm.

With the increasing incidence of cGVHD, it has become one of the most difficult complications of allo-HSCT [2]. In view of the major drawbacks of current treatments, a better therapy method is in urgent need for cGVHD. This study demonstrated therapeutic effects of oral administration of hydrogen-rich water on a scleroderma cGVHD mouse model. As to whether $\mathrm{H}_{2}$ has therapeutic effects on cGVHD through other mechanisms, further research is still needed.

\section{Data Availability}

All data relevant to the study are included in the article.

\section{Conflicts of Interest}

The authors declare no competing interest.

\section{Acknowledgments}

This work was supported by a grant from the National Natural Science Foundation of China (Grant No. 81800180) and a grant from the National Defense Science and Technology Innovation Special Zone Project-Spark Project (Grant No.20-163-00-TS-009-006-01).

\section{References}

[1] L. M. Curtis, A. Ostojic, D. J. Venzon et al., “A randomized phase 2 trial of pomalidomide in subjects failing prior therapy for chronic graft-versus-host disease," Blood, vol. 137, no. 7, pp. 896-907, 2021.

[2] P. J. Martin, "How I treat steroid-refractory acute graft-versushost disease," Blood, vol. 135, no. 19, pp. 1630-1638, 2020.
[3] S. J. Lee, G. Vogelsang, and M. E. Flowers, "Chronic graftversus-host disease," Biology of Blood and Marrow Transplantation, vol. 9, no. 4, pp. 215-233, 2003.

[4] C. Anasetti, B. R. Logan, S. J. Lee et al., "Peripheral-blood stem cells versus bone marrow from unrelated donors," The New England Journal of Medicine, vol. 367, no. 16, pp. 1487-1496, 2012.

[5] S. Sarantopoulos, A. R. Cardones, and K. M. Sullivan, "How I treat refractory chronic graft-versus-host disease," Blood, vol. 133, no. 11, pp. 1191-1200, 2019.

[6] Y. Inamoto, M. E. Flowers, B. M. Sandmaier et al., "Failure-free survival after initial systemic treatment of chronic graftversus-host disease," Blood, vol. 124, no. 8, pp. 1363-1371, 2014.

[7] L. Qian, M. Liu, J. Shen, J. Cen, and D. Zhao, "Hydrogen in patients with corticosteroid-refractory/dependent chronic graft-versus-host-disease: a single-arm, multicenter, openlabel, phase 2 trial," Frontiers in Immunology, vol. 11, article 598359, 2020.

[8] G. Socie and J. Ritz, "Current issues in chronic graft-versushost disease," Blood, vol. 124, no. 3, pp. 374-384, 2014.

[9] S. Ghobrial, C. Gonzalez, N. Yazigi et al., "Efficacy and feasibility of ruxolitinib in chronic steroid-refractory GVHD in a pediatric intestine transplant," Pediatric Transplantation, vol. 25, no. 3, article e13836, 2021.

[10] M. Schoettler, C. Duncan, L. Lehmann, E. Furutani, M. Subramaniam, and S. Margossian, "Ruxolitinib is an effective steroid sparing agent in children with steroid refractory/dependent bronchiolitis obliterans syndrome after allogenic hematopoietic cell transplantation," Bone Marrow Transplantation, vol. 54, no. 7, pp. 1158-1160, 2019.

[11] R. Zeiser, N. Polverelli, R. Ram et al., "Ruxolitinib for glucocorticoid-refractory chronic graft-versus-host disease," The New England Journal of Medicine, vol. 385, no. 3, pp. 228-238, 2021.

[12] M. E. Flowers and P. J. Martin, "How we treat chronic graftversus-host disease," Blood, vol. 125, no. 4, pp. 606-615, 2015.

[13] I. Ohsawa, M. Ishikawa, K. Takahashi et al., "Hydrogen acts as a therapeutic antioxidant by selectively reducing cytotoxic oxygen radicals," Nature Medicine, vol. 13, no. 6, pp. 688694, 2007.

[14] M. Kajiya, K. Sato, M. J. Silva et al., "Hydrogen from intestinal bacteria is protective for Concanavalin A-induced hepatitis," Biochemical and Biophysical Research Communications, vol. 386, no. 2, pp. 316-321, 2009.

[15] K. Xie, Y. Yu, Z. Zhang et al., "Hydrogen gas improves survival rate and organ damage in zymosan-induced generalized inflammation model," Shock, vol. 34, no. 5, pp. 495-501, 2010.

[16] M. Kajiya, M. J. Silva, K. Sato, K. Ouhara, and T. Kawai, "Hydrogen mediates suppression of colon inflammation induced by dextran sodium sulfate," Biochemical and Biophysical Research Communications, vol. 386, no. 1, pp. 11-15, 2009.

[17] Y. S. Yoon, M. E. Sajo, R. M. Ignacio, S. K. Kim, C. S. Kim, and K. J. Lee, "Positive effects of hydrogen water on 2, 4dinitrochlorobenzene-induced atopic dermatitis in NC/Nga mice," Biological \& Pharmaceutical Bulletin, vol. 37, no. 9, pp. 1480-1485, 2014.

[18] Z. Xu, J. Zhou, J. Cai, Z. Zhu, X. Sun, and C. Jiang, "Antiinflammation effects of hydrogen saline in LPS activated macrophages and carrageenan induced paw oedema," Journal of Inflammation, vol. 9, no. 1, p. 2, 2012. 
[19] Y. Terasaki, I. Ohsawa, M. Terasaki et al., "Hydrogen therapy attenuates irradiation-induced lung damage by reducing oxidative stress," American Journal of Physiology-Lung Cellular and Molecular Physiology, vol. 301, no. 4, pp. L415-L426, 2011.

[20] C. K. Min, "The pathophysiology of chronic graft-versus-host disease: the unveiling of an enigma," The Korean Journal of Hematology, vol. 46, no. 2, pp. 80-87, 2011.

[21] R. Zeiser and B. R. Blazar, "Pathophysiology of chronic graftversus-host disease and therapeutic targets," The New England Journal of Medicine, vol. 377, no. 26, pp. 2565-2579, 2017.

[22] L. Qian, F. Cao, J. Cui et al., "Radioprotective effect of hydrogen in cultured cells and mice," Free Radical Research, vol. 44, no. 3, pp. 275-282, 2010.

[23] L. Qian, F. Cao, J. Cui et al., "The potential cardioprotective effects of hydrogen in irradiated mice," Journal of Radiation Research, vol. 51, no. 6, pp. 741-747, 2010.

[24] Y. Chuai, J. Shen, L. Qian et al., "Hydrogen-rich saline protects spermatogenesis and hematopoiesis in irradiated BALB/C mice," Medical Science Monitor, vol. 18, no. 3, pp. BR89BR94, 2012.

[25] J. du, K. Paz, R. Flynn et al., "Pirfenidone ameliorates murine chronic GVHD through inhibition of macrophage infiltration and TGF- $\beta$ production," Blood, vol. 129 , no. 18, pp. $2570-$ 2580, 2017.

[26] K. R. Cooke, L. Kobzik, T. R. Martin et al., “An experimental model of idiopathic pneumonia syndrome after bone marrow transplantation: I. the roles of minor $\mathrm{H}$ antigens and endotoxin," Blood, vol. 88, no. 8, pp. 3230-3239, 1996.

[27] L. Qian, X. Liu, J. Shen, D. Zhao, and W. Yin, "Therapeutic effects of hydrogen on chronic graft-versus-host disease," Journal of Cellular and Molecular Medicine, vol. 21, no. 10, pp. 2627-2630, 2017.

[28] B. E. Anderson, J. M. McNiff, C. Matte, I. Athanasiadis, W. D. Shlomchik, and M. J. Shlomchik, "Recipient CD4+ T cells that survive irradiation regulate chronic graft-versus-host disease," Blood, vol. 104, no. 5, pp. 1565-1573, 2004.

[29] A. Yamamoto, E. Ashihara, Y. Nakagawa et al., "Allograft inflammatory factor-1 is overexpressed and induces fibroblast chemotaxis in the skin of sclerodermatous GVHD in a murine model," Immunology Letters, vol. 135, no. 1-2, pp. 144-150, 2011.

[30] C. J. Alves, L. P. de Santana, A. J. dos Santos et al., "Early motor and electrophysiological changes in transgenic mouse model of amyotrophic lateral sclerosis and gender differences on clinical outcome," Brain Research, vol. 1394, pp. 90-104, 2011.

[31] P. Desjardins, J. B. Hansen, and M. Allen, "Microvolume spectrophotometric and fluorometric determination of protein concentration," Current Protocols in Protein Science, vol. 55, no. 1, pp. 3-10, 2009.

[32] T. Kawamura, C. S. Huang, X. Peng et al., "The effect of donor treatment with hydrogen on lung allograft function in rats," Surgery, vol. 150, no. 2, pp. 240-249, 2011.

[33] R. Tenhunen, H. S. Marver, and R. Schmid, "The enzymatic conversion of heme to bilirubin by microsomal heme oxygenase," Proceedings of the National Academy of Sciences of the United States of America, vol. 61, no. 2, pp. 748-755, 1968.

[34] R. Gozzelino, V. Jeney, and M. P. Soares, "Mechanisms of cell protection by heme oxygenase-1," Annual Review of Pharmacology and Toxicology, vol. 50, no. 1, pp. 323-354, 2010.
[35] E. Seixas, R. Gozzelino, A. Chora et al., "Heme oxygenase-1 affords protection against noncerebral forms of severe malaria," Proceedings of the National Academy of Sciences of the United States of America, vol. 106, no. 37, pp. 1583715842, 2009.

[36] A. T. Dinkova-Kostova and P. Talalay, "NAD(P)H:quinone acceptor oxidoreductase 1 (NQO1), a multifunctional antioxidant enzyme and exceptionally versatile cytoprotector," Archives of Biochemistry and Biophysics, vol. 501, no. 1, pp. 116-123, 2010.

[37] H. Wefers, T. Komai, P. Talalay, and H. Sies, "Protection against reactive oxygen species by $\mathrm{NAD}(\mathrm{P}) \mathrm{H}$ : quinone reductase induced by the dietary antioxidant butylated hydroxyanisole (BHA). Decreased hepatic low-level chemiluminescence during quinone redox cycling," FEBS Letters, vol. 169, no. 1, pp. 63-66, 1984.

[38] S. J. Martin and D. R. Green, "Protease activation during apoptosis: death by a thousand cuts?," Cell, vol. 82 , no. 3, pp. 349352, 1995.

[39] J. H. Abraini, M. C. Gardette-Chauffour, E. Martinez, J. C. Rostain, and C. Lemaire, "Psychophysiological reactions in humans during an open sea dive to $500 \mathrm{~m}$ with a hydrogenhelium-oxygen mixture," Journal of Applied Physiology, vol. 76, no. 3, pp. 1113-1118, 1994. 